\title{
Indicadores y sistemas de cuentas ambientales y económicas integrados. Grado de instrumentación: el estado actual
}

\author{
Indicators and systems for integrated \\ environmental and economic accounts. \\ Degree of implementation: the state \\ of the art
}

Antonio Cano-Orellana acore@us.es Carmen Rodríguez-Morilla cmorilla@us.es

Universidad de Sevilla, España 


\section{Indicadores y sistemas de cuentas ambientales y económicas integrados. Grado de instrumentación: el estado actual}

\section{RESUMEN}

Los sistemas de cuentas nacionales e indicadores estándares no miden adecuadamente la calidad ambiental y el bienestar de la población. A finales de los años ochenta del pasado siglo se iniciaron reformas e iniciativas destinadas a su mejora: extensión del marco central del Sistema de Cuentas Nacionales (Naciones Unidas 1993), integración de la economía y el medio ambiente a través de un sistema de cuentas conocido como el Sistema de Contabilidad Ambiental y Económica Integrada (SCAEI) (Naciones Unidas-2003) y generación de indicadores estructurales. Cómo han evolucionado sendos aspectos y la relación entre ellos, cuál es el alcance de los cambios realizados y su aplicación en los países, es el propósito de este artículo.

Palabras claves: indicadores de sostenibilidad, sistemas de cuentas nacionales, sistema de cuentas ambientales y económicas integradas, sostenibilidad.

\section{Clasificación JEL: B41, Eo1, Q56, Q57}




\title{
Indicators and systems for integrated environmental and economic accounts. Degree of implementation: the state of the art
}

\begin{abstract}
National accounting systems and standard indicators fail as a measure of environmental quality and social well being. Since 1980 s some reforms and initiatives were launched for improvement: extending the central framework of the System of National Accounts (SNA) (United Nations, 1993), integrating the economy and the environment in a System of Integrated Environmental and Economic Accouting 2003 (SEEA-03) (United Nations, 2003) and obtaining structural indicators. The purpose of this paper is to review how both the national accounting systems and standard indicators have developed until today, its strengths and shortcomings. How they are applied to the different countries. What pattern has been observed in counting systems and indicators, and which is the relation between them.
\end{abstract}

Keywords: sustainability indicators, System of National Accounts, System of Integrated Environmental and Economic Accounts, sustainability, environmental and ecological economics.

JEL Clasification: B41, EO1, Q56, Q57

Revista de Economía, vol. XXVIII, núm. 77, julio-diciembre de 2011/77-110 

Ninguna de las facultades humanas debería ser excluida de la actividad científica. Las profundidades de la intuición, una conciencia cierta del presente, la grandeza matemática, la exactitud física, lo elevado de la razón creativa y la agudeza del entendimiento, junto a una versátil y apasionada imaginación y una delicada ternura en el mundo de los sentidos, todas ellas son esenciales para una viva y estimulante aprehensión del momento.

J. W. GOETHE

\section{INTRODUCCIÓN}

Numerosos trabajos y eventos internacionales han informado acerca del impacto de las actividades de producción y consumo sobre el medio ambiente y la calidad de vida de la población. Sin embargo, y a pesar de los avances producidos, la presión sobre los ecosistemas terrestres se ha venido agravando, así como el bienestar de amplios sectores de la población mundial. El modelo de crecimiento, animado por la globalización económica, ha reforzado estos procesos y ha desarrollado un metabolismo muy exigente con importantes requerimientos de materiales, agua y energía y una extraordinaria generación y acumulación de desechos.

La gestión del conflicto existente entre economía, medio ambiente y sociedad, a pesar de disponer de mejores herramientas y de los esfuerzos realizados en cuanto a la sensibilización y concienciación de los ciudadanos, sigue constituyendo uno de los principales retos de la realidad actual.

Una de los principales obstáculos para hacerle frente está relacionado con el paradigma científico predominante en el pensamiento económico actual, deudor de una visión mecánica y parcelaria del comportamiento económico de los individuos y de los agentes productivos que, trasladado a los sistemas contables y la generación de indicadores, se traduce en señales borrosas, cuando no inoperantes, para informarnos del estado de la sociedad y del medio ambiente, así como para orientarnos, en definitiva, en la perspectiva de la sostenibilidad hacia la consecución del desarrollo económico, social y ambiental sostenible.

Es necesario, por tanto, disponer de instrumentos analíticos que permitan evaluar la situación y dibujar los escenarios más probables, de tal forma que nos faculten para planificar estrategias y diseñar las po- 
líticas económicas y de medio ambiente más adecuadas (Morilla y Llanes, 2004b). Pero el diagnóstico que se realice estará, a su vez, muy condicionado por las herramientas contables que utilicemos a la hora de organizar y sistematizar la información económica y de medio ambiente. El deseo de disponer de un marco común consensuado en el ámbito internacional ha estimulado desde hace algún tiempo la reflexión y el debate internacional con el fin de generar propuestas que tengan que ver con el modo de incorporar los aspectos sociales y ambientales en los sistemas contables. Una primera propuesta, aunque inacabada, llegó hasta el año 1993, primera referencia en cuanto a la manera de definir, clasificar, organizar e integrar la información estadística de carácter económico y medioambiental, de manera coherente, para generar indicadores más adecuados.

Este sistema no se desarrolló casualmente, fueron muchos los planteamientos teóricos y metodológicos previamente existentes, así como la experiencia de numerosos países que ya venían trabajando en contabilidad ambiental.

El propósito de este artículo es conocer el estado de avance que han experimentado estos sistemas, no sólo en relación con su desarrollo metodológico sino respecto del grado de aplicación de estos sistemas y sus indicadores asociados. Cómo están siendo aplicados a las realidades de los diferentes países. Qué potencialidades y límites presentan los cambios realizados, cuál ha sido la pauta observada en los sistemas de cuenta y en los indicadores, así como la relación entre unos y otros.

\section{PRIMEROS INTENTOS EN LA INCORPORACIÓN DEL MEDIO AMBIENTE A LOS SISTEMAS DE CUENTAS ECONÓMICOS}

La finalidad que persigue, en general, cualquier sistema de cuentas, sin importar su índole, es proporcionar un conjunto coherente, sistemático e integrado de cuentas, balances y cuadros basados en un conjunto de conceptos, definiciones, clasificaciones y reglas contables aceptados internacionalmente.

Los primeros desarrollos de los sistemas de cuentas nacionales (en adelante SCN), tal como los conocemos actualmente, se deben a Stone (1961) y sirvieron para ir configurando sistemas de cuentas armonizados 
internacionalmente. Este proceso ha venido liderándose, a nivel institucional, por organismos como Naciones Unidas (ONU) o la Organización para la Cooperación y el Desarrollo Económico (OCDE) hasta culminar en la elaboración de manuales que utilizan la mayoría de países para organizar sus cuentas económicas y para facilitar la comparabilidad internacional: ${ }^{1}$ SCN-53, SCN-68, SCN-93 (Naciones Unidas, 1993). La última revisión de este manual se publicó en el año 2009, dando lugar al SCN-o8 (Naciones Unidas, 2009), que aún está siendo sometido a su conveniente adaptación en los distintos países. ${ }^{2}$

Estos primeros sistemas de cuentas macroeconómicas no hicieron eco en sus planteamientos de las repercusiones medioambientales que generaba la actividad económica, pues sus preocupaciones, como ya hemos apuntado, estaban más bien relacionadas con cuestiones conceptuales y con la comparabilidad de las estadísticas económicas internacionales. Además, no se presentía como un problema la escasez de los recursos naturales, que eran considerados bienes libres.

La generación de indicadores macroeconómicos obtenidos a partir de estos sistemas de cuentas se muestran claramente insuficientes (CanoOrellana, 2000) para orientarnos hacia un desarrollo que tenga en cuenta la sostenibilidad desde el punto de vista económico, ecológico y social, pues el principal recurso de los procesos productivos (la naturaleza) no fue incorporado en el análisis, así como los aspectos sociales, especialmente los relativos al bienestar de las poblaciones, cuyo tratamiento se ha mostrado claramente insatisfactorio. Son muchas las limitaciones que muestra la contabilidad nacional en este sentido, y muchas las aportaciones que se han ido generando desde los años sesenta, a partir de los debates propuestos por organismos internacionales como NU, Banco Mundial y la OCDE con la intención de mejorar nuestros sistemas contables de información.

Los intentos por mejorar los sistemas contables e incorporar el medio ambiente comenzaron en los años ochenta, a partir de los debates

${ }^{1}$ El interés oficial por la comparabilidad se remonta a 1928, cuando la Liga de las Naciones
mantuvo una Conferencia internacional sobre estadísticas económicas expresándose
el deseo de armonizar las cuentas de los diferentes países con el objetivo expreso de
"facilitar la elaboración de estimaciones del ingreso nacional a intervalos regulares"
(Naciones Unidas, 1993). no está en referencias.
${ }^{2}$ La traducción al español está a cargo de la CEPAL y se encuentra aún en estado preliminar. 
impulsados por instituciones tales como Banco Mundial o Naciones Unidas, a través del Programa de Naciones Unidas para el Medio Ambiente (PNUMA). Estos debates estuvieron, a su vez, motivados por la relevancia que el denominado desarrollo sostenible, acuñado en 1987 por la Comisión Mundial sobre el Medio Ambiente y Desarrollo, estaba alcanzando en el ámbito internacional, el cual recibió un nuevo impulso con la Conferencia de las Naciones Unidas sobre medio ambiente y desarrollo celebrada en Río de Janeiro en 1992. ${ }^{3}$

La integración de las cuestiones ambientales a los escenarios contables constituye en la actualidad un debate abordado desde distintas maneras de concebir las tensiones existentes entre economía y medio ambiente. El debate ha estado marcado, principalmente, por dos corrientes de pensamiento: la economía ambiental y la economía ecológica.

La economía ambiental centra sus análisis en el ámbito de los valores monetarios y en el análisis costes/beneficios, mientras que la economía ecológica dirige su mirada hacia el estudio de los propios recursos naturales y los ecosistemas a gestionar, -con el uso de instrumentos que generalmente vienen expresados en términos físicos-, que podría concretarse en la discusión entre sustentabilidad fuerte y débil aún presente en el marco del análisis de la sostenibilidad, también vigente en el contexto de los contenidos del trabajo de Dietz y Neumayer (2007). Asimismo, su relevancia se ve justificada por su cada vez mayor presencia en los debates de carácter institucional. $\mathrm{Al}$ respecto, es importante reseñar el hecho de que la economía ecológica (traducción en los textos en español) constituirá junto con la gobernanza los contenidos de la próxima cumbre a celebrar en Río (Río+20) en 2012 (UNEP, 2011). Con esta base teórica preliminar muchos países u organismos vinculados a los nuevos sistemas contables motivados por necesidades específicas, fueron desarrollando diferentes metodologías sobre contabilidad ambiental en ausencia de un marco común de referencia que les sirviera de apoyo. En dicho proceso Francia, Noruega, Alemania y Holanda fueron los países que se mostraron más activos en impulsar este tipo de cuentas más o menos relacionadas con las cuentas económicas. La contabilidad del patrimonio natural o las cuentas de los recursos naturales y ambientales desarrolladas en

\footnotetext{
${ }^{3}$ Ver Morilla (2004a) para un mayor desarrollo.
} 
Francia y Noruega, respectivamente, las cuentas de flujos de materiales o los balances de materia y energía aplicados en Alemania con el apoyo del Instituto Wuppertal o el Sistema NAMEA (por sus siglas en inglés) ${ }^{4}$ en Holanda, donde se integra la información ambiental y económica en un formato matricial, constituyeron, sin duda, una experiencia fundamental para que, posteriormente, tales precedentes tuvieran cabida en sistemas consensuados internacionalmente para la elaboración de cuentas ambientales.

Hay que destacar también las primeras propuestas desarrolladas en América Latina que fueron protagonizadas por países como Colombia y México. Este último, a través del Instituto Nacional de Estadística, Geografía e Informática (INEGI) y en colaboración con la oficina de estadística de las Naciones Unidas y el Banco Mundial, realizó un estudio de caso en materia de contabilidad ambiental para el año 1985, investigación que dio origen a la construcción del Sistema de Cuentas Económicas y Ecológicas de México (SCEEM) apoyado en la larga experiencia de dicha institución en el tema (INEGI, 2003).

La propuesta más novedosa, actualmente, consiste en tomar en consideración los tres núcleos con los que habitualmente se asocia el desarrollo sostenible, y combinar en un sistema matricial la contabilidad económica monetaria con la social y la medioambiental, dando lugar a lo que en la literatura se ha venido denominando como sistema de matrices de contabilidad económica y social y sus extensiones (SESAME, por sus siglas en inglés) $)^{5}$ (ver Figura 1).

\footnotetext{
${ }^{4}$ National Accounting Matrix with Environmental Accounts.

${ }^{5}$ System of Economic and Social Accounting Matrices and Extensions.
} 
Figura 1

Sistema de matrices de contabilidad económica y social y sus extensiones (SESAME)

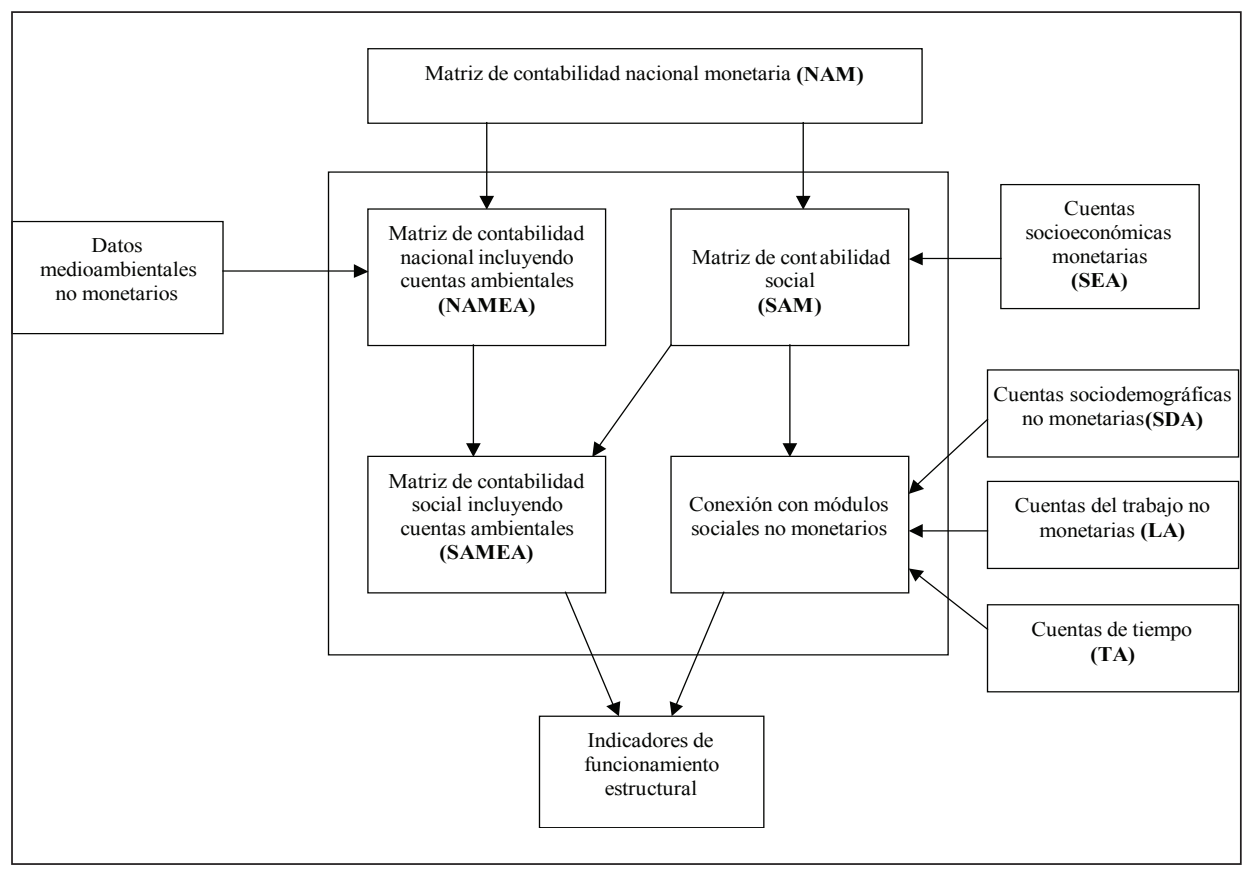

Fuente: Stahmer (2002)

La revisión del sistema de cuentas nacionales que condujo al SCN-93 constituyó una oportunidad excepcional para examinar la vinculación a este sistema de conceptos, definiciones, clasificaciones y tabulaciones de la contabilidad del medio ambiente y los recursos naturales que ya se utilizaban en algunos países, pero debido a las opiniones discrepantes acerca de las cuestiones conceptuales y prácticas no fue posible consensuar en el ámbito internacional un cambio fundamental en el núcleo central del sistema de cuentas nacionales. La incorporación de los aspectos medioambientales se hizo, por tanto, en un marco de sistema satélite del SCN como sugirieron algunos de los autores que habían intervenido en el debate (Carson, 1989; Bartelmus, 1989; Bartelmus, Stahmer y Tongren, 1991). 
Un primer paso para la integración de cuentas económicas y medioambientales lo constituyó el denominado SEEA-93, objetivo que se marcó la división estadística de la Secretaría de Naciones Unidas en atención al compromiso surgido en la Conferencia de Río en 1992. El carácter de "versión provisional" que desde un principio se atribuyó al SEEA-93 y las aplicaciones que se llevaron a cabo en los países que decidieron iniciar un sistema de cuentas ambientales y económicas integradas reflejaron las limitaciones que este sistema tenía en la práctica para su puesta en funcionamiento, lo que condujo más tarde a un proceso de revisión del manual.

El SEEA-93 sólo presentó el estado del debate sobre cómo introducir la medición del medio ambiente en los sistemas de cuentas nacionales, y dejó muy abierta la metodología concreta a utilizar. Debido a ello se constituyó en 1994, en el seno de la comisión estadística de la ONU, el denominado Grupo de Londres sobre contabilidad ambiental, ${ }^{6}$ que ha sido el que ha dinamizado el debate internacional en torno a este tema y al proceso de revisión del SEEA, que finalizó en el año 2003 con la publicación del nuevo marco contable SEEA-03.

El Grupo de Londres se creó con los siguientes objetivos: a) proporcionar un foro anual para que los principales países y organizaciones internacionales pudieran intercambiar conocimientos prácticos y teóricos sobre la elaboración de cuentas ambientales vinculadas al Sistema de cuentas nacionales, b) poner a disposición del público en general, en forma impresa o electrónica, los materiales presentados para el debate por esos países y organizaciones, y c) desempeñar una función de liderazgo en la definición de normas internacionales relacionadas con la teoría y la práctica de la contabilidad ambiental.

Hasta 1997 el Grupo de Londres sirvió, fundamentalmente, de foro para el intercambio de información sobre experiencias nacionales en la compilación de cuentas ambientales, pero en ese mismo año la comisión de estadística propuso al Grupo que iniciara una revisión del SEEA-93, labor que venía desempeñando hasta 2003, año en el que se publicó el

${ }^{6}$ El Grupo de Londres es un grupo de expertos que provienen, principalmente, de organismos nacionales e internacionales de estadística. Aunque se creó en el año 1993, su primera reunión tuvo lugar hasta 1994 en la ciudad de Londres (de ahí el nombre). Sus reuniones anuales constituyen un foro para el examen, comparación y discusión de los trabajos en curso por los participantes hacia el desarrollo de cuentas ambientales. 
nuevo Manual de contabilidad ambiental y económica integrada (SEEo3), como resultado de varias reuniones del Grupo de Londres, su comité de coordinación y de un amplio proceso de consultas.

Este manual recoge de forma sistemática y coherente las precisiones y delimitaciones conceptuales referentes a la contabilización de flujos físicos vinculados a la esfera medioambiental y su conexión con flujos monetarios asociados con las actividades de producción y consumo. Se estructura con base en las siguientes cuentas: 1) cuentas de flujos físicos [capítulo 3], 2) cuentas de flujos híbridos [capítulo 4 y 6], 3) cuentas de gastos en protección medioambiental y gestión de recursos [capítulos 5 y 6], 4) cuentas de activos de recursos naturales [capítulo 7], y 5) agregados ajustados [capítulos 9 y 10].

\section{EL ESTADO ACTUAL Y LOS AVANCES LOGRADOS A PARTIR DE 2003}

El Grupo de Londres sobre contabilidad ambiental ha jugado en los últimos años un papel destacado en el avance de las metodologías de contabilidad ambiental y económica, así como en proporcionar un foro para compartir experiencias nacionales e internacionales en este campo. Los logros más notables han sido su contribución al Manual de cuentas económicas y ambientales (SEEA-2003) y, más recientemente, a la consolidación del Sistema de Contabilidad Ambiental y Económica para el Agua, SEEAW (por sus siglas en inglés), que fue aprobado en el trigésimo periodo de sesiones de la comisión de estadística de Naciones Unidas como una guía de referencia internacional en la aplicación de los distintos países.

A partir de 2003 la estrategia del Grupo ha estado basada en tres objetivos principales: 1) preparar manuales que proporcionen directrices metodológicas y prácticas para la aplicación de componentes seleccionados del SEEA-03 (por ejemplo, contabilidad de los recursos hídricos y minerales), 2) continuación del desarrollo teórico y práctico de los componentes de la contabilidad ambiental en los cuales aún no se ha avanzado, y 3) prestar asistencia para promover la aplicación del SEEA-03 en los países y utilizar las cuentas ambientales en el contexto de los debates sobre políticas, en los planos nacional e internacional. En particular, la intención de que 
las cuentas se integren mejor con las actividades actuales de recopilación de datos ambientales y elaboración de indicadores, y que se utilicen más frecuentemente en el proceso normativo.

El modus operandi elegido fue la creación de grupos de trabajo sobre temas seleccionados. En el $8^{\circ}$ encuentro celebrado en noviembre de 2003 en Roma, se establecieron cuatro grupos de trabajo: (i) contabilidad de los recursos hídricos, moderado por la división de estadística de Naciones Unidas, (ii) contabilidad de los recursos energéticos y minerales, moderado por Dinamarca, (iii) usos políticos de las cuentas ambientales, moderado por Suecia y la Agencia europea de medio ambiente y (iv) formas de introducir las dimensiones sociales en las cuentas ambientales, como base para contribuir a la evaluación de la sostenibilidad, moderado por Suecia. En respuesta a la petición de muchos países para integrar las estadísticas del medio ambiente en su contabilidad económica, y elevar en importancia el perfil de estas cuentas, se creó en 2005 el comité de expertos en cuentas económicas y medioambientales de Naciones Unidas, UNCEEA (por sus siglas en inglés), cuyos objetivos eran: (a) difundir la contabilidad ambiental y económica y sus estadísticas conexas, (b) elevar el SEEA a un nivel de norma internacional, y (c) promover su aplicación en los diferentes países.

Con el fin de lograr los objetivos mencionados, se encomendaron las siguientes tareas a dicho comité: (i) coordinación, (ii) promoción de las cuentas, (iii) avanzar en la metodología, y (iv) armonización.

\section{Cuadro 1}

Avance en la integración de cuentas económicas y ambientales

\begin{tabular}{|l|l|l|}
\hline 1993 & SEEA-93 & $\begin{array}{l}\text { Primer borrador sobre integración de } \\
\text { cuentas ambientales y económicas }\end{array}$ \\
\hline $\mathbf{1 9 9 4}$ & $\begin{array}{l}\text { Creación del Grupo de } \\
\text { Londres }\end{array}$ & $\begin{array}{l}\text { Formado por países y organismos con el } \\
\text { objetivo de establecer un foro de discusión } \\
\text { en la instrumentación de las cuentas }\end{array}$ \\
\hline $\mathbf{1 9 9 7}$ & $\begin{array}{l}\text { Se inicia la revisión del } \\
\text { SEEA-93 }\end{array}$ & $\begin{array}{l}\text { Através de encuentros anuales se estableció } \\
\text { un proceso de discusión que finalizó en } \\
2002\end{array}$ \\
\hline
\end{tabular}




\begin{tabular}{|c|c|c|}
\hline 2001 & SEEA-OO & $\begin{array}{l}\text { La UNSD, con el apoyo del grupo de } \\
\text { Nairobi, publica un manual operativo que } \\
\text { sirva de guía a los países. También se recoge } \\
\text { la experiencia desde } 1993 \text { en la elaboración } \\
\text { de cuentas }\end{array}$ \\
\hline \multirow[t]{4}{*}{2003} & SEEA-03 & Publicado por Naciones Unidas, el FMI, \\
\hline & $\begin{array}{l}\text { Creación del subgrupo } \\
\text { contabilidad de agua }\end{array}$ & $\begin{array}{l}\text { En el } 8^{\circ} \text { encuentro del Grupo de Londres } \\
\text { celebrado en Roma con el objetivo de } \\
\text { elaborar un SEEA-Agua (SEEA-W por sus } \\
\text { siglas en inglés) y armonizar las estadísticas } \\
\text { de este recurso }\end{array}$ \\
\hline & $\begin{array}{l}\text { Creación del subgrupo } \\
\text { de cuentas minerales y } \\
\text { energéticas }\end{array}$ & $\begin{array}{l}\text { Con el objetivo de armonizar a nivel } \\
\text { internacional dichas cuentas. Trabaja } \\
\text { en colaboración con el grupo de Oslo en } \\
\text { estadísticas energéticas }\end{array}$ \\
\hline & $\begin{array}{l}\text { Creación del subgrupo } \\
\text { sobre la ampliación del } \\
\text { SEEA a los aspectos } \\
\text { sociales }\end{array}$ & $\begin{array}{l}\text { Conexión con cuentas del trabajo, usos del } \\
\text { tiempo, hogares }\end{array}$ \\
\hline 2004 & SEEA-F-O3 & $\begin{array}{l}\text { Se publica el manual que proporciona una } \\
\text { guía para integrar las cuentas económicas } \\
\text { y medioambientales referidas a la pesca, } \\
\text { elaborado por la división estadística de NU } \\
\text { y la FAO }\end{array}$ \\
\hline \multirow[t]{2}{*}{2005} & Creación de la UNCEEA & $\begin{array}{l}\text { En el seno de la Comisión estadística de } \\
\text { NU se crea un Comité de expertos en } \\
\text { cuentas económicas y medioambientales } \\
\text { con el objetivo de coordinar, instrumentar, } \\
\text { revisar y armonizar }\end{array}$ \\
\hline & $\begin{array}{l}\text { Creación del Grupo de } \\
\text { Oslo }\end{array}$ & $\begin{array}{l}\text { Con el objetivo de la armonización } \\
\text { internacional de las estadísticas de energía }\end{array}$ \\
\hline \multirow[t]{2}{*}{2007} & Borrador SEEA-W & $\begin{array}{l}\text { Se adopta como una guía estadística } \\
\text { internacional }\end{array}$ \\
\hline & $\begin{array}{l}\text { Se inicia la revisión del } \\
\text { SEEA }\end{array}$ & $\begin{array}{l}\text { La revisión se aborda por temas específicos: } \\
\text { agua, energía, pesca, flujo de materiales. } \\
\text { Espera publicarse en } 2012\end{array}$ \\
\hline 2008 & $\begin{array}{l}\text { Ampliación de la } \\
\text { UNCEEA } r \text { hacia } \\
\text { las } \quad \text { estadísticas } \\
\text { medioambientales }\end{array}$ & $\begin{array}{l}\text { En respuesta a la petición de la conferencia } \\
\text { de estadísticos europeos y de la conferencia } \\
\text { sobre cambio climático y estadísticas } \\
\text { oficiales, amplia sus competencias hacia las } \\
\text { estadísticas del medio ambiente, incluidas } \\
\text { las estadísticas sobre cambio climático }\end{array}$ \\
\hline
\end{tabular}




\section{REVISIÓN ACTUAL DEL SEEA-O3}

Actualmente el SEEA-03 está en un proceso de revisión que inició en 2007. La intención del nuevo SEEA es que esté diseñado para el análisis, la toma de decisiones y la formulación de políticas, sea cual sea la estructura industrial o etapa de desarrollo alcanzado por el país que lo aplique. Es decir, que proporcione un marco que pueda utilizarse para evaluar el desarrollo sostenible y elaborar indicadores al respecto. La gestión y supervisión está siendo realizada por el UNCEEA, aunque el Grupo de Londres sobre contabilidad ambiental juega un papel importante en cuanto al apoyo técnico.

El SEEA-03 quedó incompleto en muchos temas específicos, por lo que la revisión está teniendo lugar tanto a nivel general de marco contable como a nivel particular. (i) SEEA-W, proporciona un marco coherente para organizar la información sobre el agua y sus relaciones con el sistema de producción y consumo. La comisión estadística de NU lo aprobó como norma estadística internacional en 2007, y alentó su instrumentación en los países. Las Recomendaciones internacionales para las estadísticas del agua, IRWS (por sus siglas en inglés), suministran el apoyo para armonizar las estadísticas del agua internacionalmente, para la aplicación de la SEEA-W, proporciona, además, la información necesaria para derivar indicadores coherentes y consistentes en el tiempo. Se esperaba que en 2010 finalizase el manual sobre recomendaciones en la compilación de estadísticas de agua. (ii) SEEA-E proporciona el marco para integrar las cuentas de la energía y la cuenta de emisiones a la atmósfera, incorpora también los balances de energía. La revisión sigue la Recomendación internacional de estadísticas de energía, IRES (por sus siglas en inglés), que cubre una amplia gama de cuestiones relativas a los conceptos básicos, definiciones y clasificaciones, recopilación de datos, balances de energía y su difusión. El sistema está apoyado por el Grupo de Oslo sobre estadísticas energéticas. Estaba prevista su finalización para 2011 (iii) SEEA-MFA: el Sistema de contabilidad ambiental y económica para flujos de materiales está siendo abordado por el Grupo de Londres, Eurostat ${ }^{7}$ y

\footnotetext{
${ }^{7}$ Eurostat publicó en 2001 una guía para la contabilidad de flujos de materiales, Economywide material flow accounts and derived indicators. A methodological guide, aunque se pretende que haya un manual y evitar de este modo la confusión entre los usuarios de los manuales.
} 
el grupo de la OCDE sobre flujos de materiales. Se trata de ampliar aspectos que quedaron pendientes, proporcionando un marco contable relativo a la extracción nacional, importaciones y exportaciones, el suministro y uso de productos y la generación de residuos valorados en unidades físicas. Las cuentas de flujo de materiales, en principio, intentan cubrir todos los flujos de materiales, incluidos los productos energéticos y el agua, de tal manera que los tres subsistemas sean coherentes en términos de conceptos, definiciones y clasificaciones. Estaba prevista su aprobación para el año 2011. (iv) SEEA-F: en 2005 el manual de contabilidad ambiental y económica para la pesca fue publicado conjuntamente por la división de estadística de NU y el departamento de pesca de la FAO. El Manual es una guía de buenas prácticas y proporciona directrices sobre la contabilidad ambiental y económica para la pesca de captura y la acuicultura, está ilustrado con ejemplos para la elaboración de las cuentas en los distintos países.

En un principio estaba previsto que el SEEA completo se publicara en el año 2010, pero después de una evaluación más detenida del programa de trabajo y de la financiación del proyecto se llegó a la conclusión de que la publicación debería aplazarse a 2012. La publicación constará de tres volúmenes: 1) se referirá a la norma estadística internacional, 2) abarcará los temas en los que no se pudo llegar a un consenso, pero que son altamente pertinentes para la política, y 3) contendrá las aplicaciones de las cuentas presentadas en los volúmenes 1 y 2.

\section{ESTADO ACTUAL DE APLICACIÓN DE LA CONTABILIDAD ECONÓMICA Y AMBIENTAL}

La división de estadística de Naciones Unidas llevó a cabo en 2006 una evaluación mundial de las estadísticas del medio ambiente y la contabilidad ambiental y económica, bajo los auspicios del comité de expertos (UNCEEA). La evaluación puso de relieve el estado actual de la aplicación nacional de los programas de estadísticas del medio ambiente y de contabilidad ambiental y económica en los países, señala los factores que impedían su desarrollo y pone de manifiesto las prioridades en el futuro. Se llevó a cabo en dos fases a través de cuestionarios enviados a 192 oficinas 
nacionales de estadística de los países miembros y a 11 bancos centrales (en el caso de países en el que los programas de cuentas nacionales incumben a los bancos centrales).

La fase I se inició en diciembre de 2006 y abarca la información general sobre los programas de estadísticas del medio ambiente y de contabilidad ambiental y económica. ${ }^{8}$ Incluye cuestiones relativas a la estructura institucional, los temas, los obstáculos con que se ha tropezado en el desarrollo y la aplicación de los programas y los planes de los países para el futuro. La fase II (aún en marcha) girará en torno de cuestionarios de seguimiento basados en temas concretos identificados en la fase I de la evaluación. Los cuestionarios se enviaron durante el primer semestre de 2007 únicamente a los países que hubieran indicado que realizaban actividades respecto de un tema concreto.

Los resultados de la PRIMERA FASE pueden sintetizarse como sigue (ver cuadro 2): (i) prácticamente todos los países que respondieron cuentan con un programa de estadísticas ambientales. La distribución geográfica del programa abarca desde un máximo de 100\% de los países de Europa y América del Norte hasta un mínimo de $75 \%$ de los países de Asia Occidental; (ii) aunque los programas de estadísticas ambientales en general están diseminados entre diversas instituciones, existen acuerdos oficiales $\mathrm{u}$ oficiosos de intercambio de datos para la cooperación entre las instituciones; (iii) en las oficinas nacionales de estadística los programas de estadísticas del medio ambiente suelen encontrarse en dependencias separadas; (iv) casi la mitad de los que respondieron a la evaluación, aunque desigualmente, cuentan con un programa de contabilidad ambiental y económica; (v) el programa de contabilidad ambiental y económica suele encontrarse en la institución que se encarga de recopilar las cuentas nacionales, ya sean las oficinas nacionales de estadística o los bancos centrales, y, en algunos casos, su recopilación corresponde al Ministerio de Medio Ambiente; (vi) los programas de estadísticas del medio ambiente y de contabilidad ambiental y económica están integrados, prácticamente, en

\footnotetext{
${ }^{8}$ Para efectos de la evaluación, se entiende por programa de estadísticas del medio ambiente un programa amplio (aunque no sea exhaustivo en cuanto a los temas que abarca) que facilite información sobre el medio ambiente, y no sobre temas concretos como suelen hacer los ministerios competentes para efecto de la gestión de recursos. Un programa de contabilidad ambiental y económica prevé la compilación de cualquier parte/módulo de las cuentas en términos físicos o monetarios.
} 
la mitad de los países, lo que significa que en ambos programas se utilizan las mismas definiciones, clasificaciones y recopilaciones de datos; (vii) de hecho casi todas las instituciones que recopilan estadísticas del medio ambiente o cuentas ambientales y económicas tienen prevista la continuidad y ampliación del actual programa de recopilación con la incorporación de los recursos hídricos, energía, emisiones a la atmósfera, recursos forestales y tierras, y ecosistemas, cuentas de flujo de materiales; aunque esta última sólo supone una cuestión prioritaria en los países europeos, y (viii) los países señalaron la falta de recursos humanos y financieros como el factor más común que obstaculiza el desarrollo de ambos programas. En relación con la recopilación se destacó la disponibilidad y la calidad de los datos. La evaluación demostró que aproximadamente en la mitad de los países los programas han sido reforzados con actividades de asistencia técnica y fondos procedentes principalmente de organizaciones internacionales, algunos reciben apoyo y fondos de proyectos bilaterales de cooperación y cerca de la mitad de los países tuvieron asistencia financiera de Eurostat.

\section{Cuadro 2}

Programas de estadísticas de medio ambiente y de contabilidad ambiental y ecnonómica en los países

\begin{tabular}{|c|c|c|c|c|c|c|c|}
\hline & \multirow[b]{2}{*}{$\begin{array}{r}\text { Número de } \\
\text { países }\end{array}$} & \multicolumn{3}{|c|}{ Estadísticas del medio ambiente } & \multicolumn{3}{|c|}{ Contabilidad ambiental y económica } \\
\hline & & $\begin{array}{r}\text { Número de } \\
\text { países que } \\
\text { respondieron a } \\
\text { la parte } \mathrm{A}\end{array}$ & $\begin{array}{r}\text { Número de } \\
\text { países que } \\
\text { cuentan con } \\
\text { un programa }\end{array}$ & $\begin{array}{r}\text { Porcentaje de } \\
\text { países que } \\
\text { enviaron } \\
\text { respuestas }\end{array}$ & $\begin{array}{r}\text { Número de } \\
\text { países que } \\
\text { respondieron } \\
\text { a la parte B }\end{array}$ & $\begin{array}{r}\text { Número de } \\
\text { países que } \\
\text { cuentan con un } \\
\text { programa }\end{array}$ & $\begin{array}{r}\text { Porcentaje de } \\
\text { países que } \\
\text { enviaron } \\
\text { respuesta }\end{array}$ \\
\hline & 1) & 2) & 3) & 4) $=3) / 2)$ & 5) & 6) & 7) $=6) / 5)$ \\
\hline Grupos económicos & & & & & & & \\
\hline Países desarrollados & 37 & 26 & 26 & 100 & 27 & 23 & 85 \\
\hline Países con economías en transición & 19 & 10 & 10 & 100 & 10 & 3 & 30 \\
\hline Países en desarrollo & 136 & 48 & 41 & 85 & 50 & 17 & 34 \\
\hline Grupos geográficos & & & & & & & \\
\hline Oceanía & 14 & 6 & 5 & 83 & 6 & 4 & 67 \\
\hline América Latina y el Caribe & 33 & 16 & 15 & 94 & 16 & 4 & 25 \\
\hline América del Norte & 2 & 1 & 1 & 100 & 2 & 1 & 50 \\
\hline Europa & 43 & 28 & 28 & 100 & 29 & 21 & 72 \\
\hline
\end{tabular}

Nota: la fase I de la evaluació mundial está integrada por dos partes: parte A sobre estadísticas del medio ambiente y parte B sobre contabilidad

ambiental y económica. No todos los países respondieron a ambas partes. Por ello, las columnas 2) y 5) son distintas. 
Por lo que se refiere a la FASE II, la evaluación realizada hasta el momento atañe a dos temas específicos: las estadísticas y cuentas de recursos hídricos y las cuentas de energía. Respecto a los primeros: (i) abarcan una amplia gama de datos que son reunidos y recopilados por diferentes organismos en países de todo el mundo. Como diversos organismos reúnen y recopilan datos sobre el agua para sus propios fines, a fin de extraer indicadores específicos del sector (por ejemplo, los organismos agrícolas acopian información sobre el agua para el riego, los ministerios de recursos hídricos lo hacen para establecer balances hídricos, etc.), comúnmente se suelen observar diferencias y superposiciones en el sistema de información sobre ese recurso. Además, otro aspecto importante, la terminología, definiciones y clasificaciones utilizadas parecen diferir entre los organismos y no se ajustan a las normas de estadística internacionales (por ejemplo, la Clasificación Industrial Internacional Uniforme de todas las Actividades Económicas (CIIU) y la Clasificación Central de Productos (CCP)); (ii) la necesidad de información sobre los recursos hídricos para sustentar una gestión integrada de esos recursos ha contribuido a que se incorporen, cada vez más, las estadísticas y cuentas de los recursos hídricos como elemento en los sistemas estadísticos nacionales de los países desarrollados y en desarrollo; (iii) setenta países indicaron que tienen un programa ordinario de estadísticas de los recursos hídricos, 33 comunicaron que aplican un programa ordinario de contabilidad del agua y otros 11 señalaron que tenían la intención de comenzar un programa de ese tipo en los próximos dos años; (iv) la evaluación demostró, claramente, que la integración de las estadísticas del agua plantea problemas. Las oficinas nacionales de estadísticas deben contribuir a congregar a las diversas partes interesadas para que conciban un sistema de información polivalente común. De esta manera el sistema aseguraría que los datos se recopilen una sola vez y se utilizen varias veces sobre la base de conceptos, definiciones y clasificaciones aceptadas de forma generalizada.

En cuanto a las cuentas de energía: (i) éstas ocupan un lugar destacado en el programa estadístico. En la Evaluación mundial de estadísticas de energía, 99 países indicaron que cuentan con un programa de estadísticas de energía, un número cada vez mayor de países compilan las cuentas sobre activos o corrientes de energía como parte de su programa 
de trabajo ordinario. (ii) La evaluación mundial determinó que la falta de una metodología convenida era uno de los obstáculos más apremiantes a la ejecución de las cuentas de energía. Los métodos utilizados en la ejecución de esas cuentas y las definiciones y clasificaciones de los recursos y productos energéticos, así como su valoración difieren mucho entre los países.

\subsection{El estado actual de aplicación de la contabilidad ambiental en América Latina}

Si bien es cierto que a comienzos de los años noventa varios países, auspiciados por organismos internacionales, elaboraron proyectos de cuentas ambientales, éstas han tenido un desarrollo parcial y desigual. La mayoría de ellos no continuaron desarrollándose, bien por falta de capacidad técnica o por no disponer de la financiación necesaria. Sin embargo, el tema ha sido revitalizado en los últimos años y algunos países han comenzado a elaborar planes para instrumentar en el futuro próximo un sistema de cuentas satélites del medio ambiente.

El cuadro 3 resume ampliamente el estado de avance en la consolidación de este tipo de cuentas elaborado a partir de una encuesta sobre cuentas ambientales realizada entre septiembre-octubre de 2003 por la CEPAL (2005), en la que se preguntó por el estado de avance de los países en la elaboración de sus cuentas ambientales, los proyectos en procesos y los planes futuros para su desarrollo. 


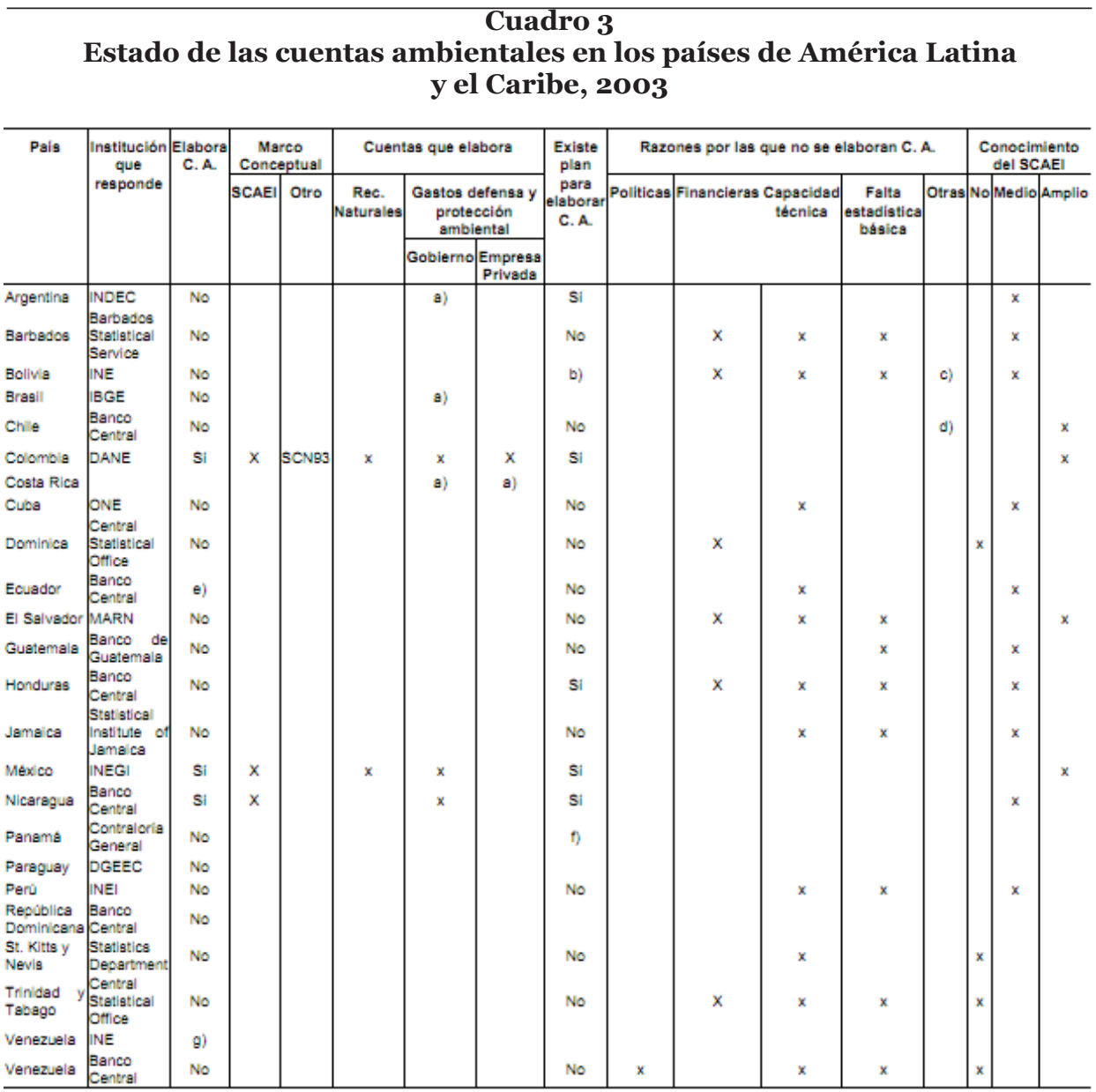

Fuente: CEPAL (2005): 59.

Sin lugar a dudas, en todo este proceso resaltan claramente los logros conseguidos en México por el INEGI (2010) que en septiembre de 2010 publicó su segunda versión de las Cuentas económicas y ecológicas de México, 2003-2008 ${ }^{9}$ en valores corrientes, con el objetivo de identificar el

${ }^{9}$ La primera versión se elaboró para el periodo 1999-2004 y estuvo encaminada a obtener un indicador, el Producto Interno Neto ajustado ambientalmente (PINE), lo que enriqueció la base informativa sobre la que se sustenta el conocimiento del desarrollo económico y ambiental del país INEGI (2006). 
impacto ambiental de las actividades económicas y cuantificar el agotamiento de los recursos naturales y la degradación del medio ambiente; así como el gasto que la sociedad efectúa para resarcir los daños ambientales consecuencia del proceso productivo de bienes y servicios. Junto a México, Colombia es otro de los países que más han avanzado en el desarrollo de sus cuentas ambientales, a través del Departamento Administrativo Nacional de Estadística (DANE). El Sistema de Contabilidad Económico-Ambiental Integrado de Colombia, COLSCEA, responde a la modalidad de cuentas satélites integradas a las cuentas nacionales, que permiten ampliar la descripción de un tema concreto como salud, educación, turismo y medio ambiente, mediante los cuadros e indicadores correspondientes a cada caso. Sus cuentas ambientales abarcan las cuentas del gasto en protección ambiental y las cuentas físicas de activos naturales (petróleo, gas natural, hierro...).

\section{LA NECESIDAD DE INDICADORES AMBIENTALES: INDICADORES DE SÍNTESIS, SU CONEXIÓN CON LOS SISTEMAS DE CUENTAS NACIONALES}

A continuación reflexionaremos en torno de la necesidad de proveer las cuentas nacionales con indicadores adecuados a las nuevas necesidades, a señalar someramente qué papel pueden jugar los indicadores ambientales en cuanto a suplir las limitaciones que los indicadores al uso presentan, qué avances se han producido al respecto, y su función en relación con las propuestas de mejora de las cuentas nacionales, con la emisión de señales correctas que modifiquen las tendencias erróneas y con el diseño de instrumentos imprescindibles en el marco de los procesos evaluativos.

Los indicadores ambientales surgen, entre otras razones, para intentar paliar las limitaciones de los indicadores económicos estándares, tales como el PIB, VAB o RN, para dar cuenta tanto de la escala de la actividad económica y sus efectos sobre el conjunto de los ecosistemas, como de la contribución de esta al bienestar de la sociedad. ${ }^{10}$ Existe, en la actualidad, un

${ }^{10} \mathrm{Al}$ respecto es conveniente reconocer la importancia de la iniciativa de Naciones Unidas de la Evaluación de los ecosistemas del milenio, para examinar, entre otros, el impacto de la actividad económica en los ecosistemas desde el punto de vista de garantizar, no sólo los objetivos del milenio, sino el bienestar futuro de la población mundial.United Nation (2005) http://www.maweb.org/en/Global.aspx 
nivel de acuerdo suficientemente amplio en el que, aunque los indicadores ya referidos han cumplido - y para algunos aspectos cumplen aún- una importante función, se muestran profundamente insuficientes para proporcionar una imagen fiel de la complejidad económica actual, así como de la salud de los ecosistemas y del bienestar de la sociedad (Stiglitz, Sen y Fitoussi, 2011).

En efecto, los indicadores ambientales, generalmente expresados en unidades físicas, vienen a satisfacer aquellos aspectos que han quedado fuera del circuito convencional de valoración económica. Constituyen, además, una valiosa herramienta para cumplir, e incluso discutir, los resultados que, por lo general, se consiguen utilizando exclusivamente magnitudes monetarias (Cano-Orellana, 2009).

$\mathrm{Al}$ llegar a este punto, resulta conveniente -como sugieren Azqueta et al. (2004) - plantearse una serie de interrogantes, preguntas relevantes a diferentes niveles, que justifiquen el cambio conceptual, pero sobre todo metodológico, que subyace tras las críticas realizadas a las magnitudes macroeconómicas usadas por la economía estándar y los indicadores asociados. De un lado, sería útil valorar si merece la pena el esfuerzo contable que supone modificar sustancialmente la manera en que se construye la contabilidad actual por los diferentes países. De otro, es necesario intentar dar respuestas a preguntas tales como: ¿qué pretendemos medir?, ¿cómo hacerlo?, ¿̇cuándo?, ¿dónde medir? Por último, ¿̇cómo resolver el problema de carencia de información significativa?

El principal objetivo de las cuentas nacionales consiste en estimar una medida agregada de la actividad económica, operada a través del mecanismo de mercado. Las magnitudes macroeconómicas de la contabilidad nacional son una herramienta fundamental en el diagnóstico sobre el estado de la economía, sirven de soporte para la toma de decisiones tanto públicas como privadas (Azqueta et al., 2004).

En la actualidad, la contabilidad nacional, como se ha comentado al principio de este artículo, se centra fundamentalmente, por una parte, en dar cuenta de cuál es el estado de la producción de bienes y servicios y la relación entre ellos y, por otra, de su viabilidad futura. Sin embargo, se asume de manera amplia que su ámbito de aplicación es muy limitado, no permite abarcar el conjunto de exigencias propias de las sociedades actuales. En correspondencia con lo anterior, la adaptación de las cuentas 
nacionales a las nuevas recomendaciones, establecidas internacionalmente, plantea la necesidad de ampliar el campo de la información, de los datos a gestionar, de los indicadores a desarrollar.

La revisión puede abordarse, como sugieren Azqueta et al. (2004), desde dos puntos de vista: a) la renta como un nivel agregado de gasto, como la conciben, y b) la renta como medida del bienestar, como se definió desde la economía del bienestar o del equilibrio general. O también se puede optar por una perspectiva más amplia e intentar superar el corsé que imponen las constricciones conceptuales y metodológicas de la economía estándar, buscar nuevas vías, como ya se ha puesto de manifiesto. Ya que “...nada es más irracional que un sistema de contabilidad incompleto...” (Kapp, 2006: 58). Si bien es cierto que la asociación de riqueza monetaria y bienestar social, ampliamente aceptada, es un logro que han conseguido los indicadores monetarios y aún no los indicadores ambientales.

En la actualidad existen centenares de indicadores ambientales. Abordan, por lo general, aspectos parciales (agua, contaminación atmosférica, agricultura, cambio climático, etc.) El hecho de ser tantos y tan específicos hacen que su manejo resulte difícil y poco eficaz. Por ello, es importante reconocer el esfuerzo de Naciones Unidas, de la OCDE, de la Agencia Europea del Medio Ambiente, del Ministerio de Medio Ambiente, tal vez el organismo más comprometido con la información ambiental, o particularmente en España, que se ha traducido en la elaboración de la serie Sistema español de indicadores ambientales, también agrupándolos en áreas tales como: agua y suelo, atmósfera y residuos, costas y medio marítimo, medio urbano, y turismo. $\mathrm{O}$ el Banco Público de Indicadores Ambientales -BPIA- (aunque sólo disponga de información sobre energía: consumo de energía primaria; eco-eficiencia del sector energético; intensidad de $\mathrm{CO}_{2}$ de origen energético e intensidad de energía primaria).

Junto a los indicadores anteriores, con el afán de encontrar indicadores sintéticos, se han diseñado algunos de ellos: la huella ecológica, desarrollada por M. Wackernagel y W. Rees a principios de los años noventa del pasado siglo; la intensidad de material por unidad de servicio o de producto (MIPS o MIPO); las mochilas ecológicas, construidas por Schmidt-Bleek en 1994 (Instituto Wuppertal); los niveles de cargas críticas, diseñado por la Comisión económica de Naciones Unidas para Europa; el índice de bienestar económico sostenido (ISEW), creado por H. E. Daly, 
J. Cobb y C. Cobb; el genuino índice de progreso (GPI); el Redefining progress; entre otros (Cano-Orellana, 2000). Todos ellos surgen en un contexto donde la necesidad de nuevas herramientas (Environment Canada, 1991, OCDE, 1993, 1994) se hace cada vez más urgente para -como pone de manifiesto el informe de 1996 del Programa para el Medio Ambiente de Naciones Unidas- "evaluar y vigilar la evolución y tendencias en el estado del medio ambiente, el uso de los recursos naturales y los procesos de desarrollo" (Rodenburg, 1992).

$\mathrm{Al}$ ser necesaria la cuantificación en el análisis de la sostenibilidad y en la ampliación del marco contable, la inconmensurabilidad que presentan determinadas magnitudes no debe impedir avanzar en un marco más explicativo de la realidad y de orientación para el desempeño de buenas prácticas. Así lo entendía Kapp respecto del análisis de los costes sociales: "La cuantificación y medida tiene, sin duda, su lugar tanto en el análisis científico como en la formulación de política. Pero en muchos casos el esfuerzo para cuantificar algo puede ser vano. Es más, podemos crear la «apariencia» de una medida cuantitativa cuando, de hecho, no hacemos nada de eso" (2006: 63).

De hecho, la utilidad de los indicadores ambientales va más allá de su exacta cuantificación. Es más, como el mismo autor afirma: "de los conceptos de 'valor social' y de 'evaluación social' surgen los más importantes problemas, hasta ahora no resueltos por la ciencia económica” (Kapp, 2006: 64).

Es por ello, y a pesar de ello, que, "a la vista del alcance del deterioro del medio ambiente no parece haber nada más importante que el desarrollar indicadores fiables para valorar, medir y evaluar, en la mayor medida posible, el grado y las consecuencias de este deterioro en sus diversas manifestaciones" (Kapp, 1995a: 139). Asumamos que los indicadores ambientales, como indicadores sociales,

...deben su origen a la creciente conciencia de que los indicadores económicos, expresados en términos monetarios, son inadecuados y no miden, ni son capaces de expresar de forma adecuada, lo que ocurre en la esfera económica y social al ignorar e incluso ocultar las importantes consecuencias negativas del proceso económico, es decir, omiten los costes sociales reflejados en deterioro del medio ambiente humano en el sentido físico y social del término... (Kapp, 1995b:205). 
En 1995 el Instituto de Recursos Mundiales (WRI, por sus siglas en inglés) estableció criterios para la construcción de indicadores ambientales y precisa que aunque "los indicadores representan un modelo empírico de la realidad, no la realidad en sí misma" (Cano-Orellana, 2008) son muy necesarios. Sostiene, además, que "en aras a favorecer su manejo para orientar políticas públicas y facilitar la participación de la ciudadanía, estos indicadores han de ser necesariamente comprensibles" (Cano-Orellana, 2008). Algunas de las características que deberían verificar los indicadores para este propósito serían: (i) utilidad para el usuario: los indicadores deben ser relevantes y comprensibles para los profanos; (ii) relevantes para las políticas: los indicadores deben ajustarse a interpretar el estado del ambiente y las presiones de la actividad humana en relación con los objetivos de políticas y programas estatales, regionales y locales; (iii) elevada capacidad de síntesis: los indicadores deben ser capaces de sintetizar en un valor numérico una gran cantidad de información.

La necesidad de construir indicadores ambientales se justifica, principalmente, por la necesidad de disponer de información adecuada y manejable que oriente hacia la acción. No pueden ser concebidos como herramientas técnicas que sólo interesen a los técnicos. Por el contrario, han de servir para favorecer y garantizar la gestión y planificación de la sostenibilidad, en cuanto a la participación activa de la población en la toma de decisiones públicas. Quisiéramos traer a colación unas reflexiones vertidas en el Informe de recursos mundiales, 2004 del WRI. En su prólogo, Cristina Narbona, titular del Ministerio de Medio Ambiente español en ese tiempo, afirmaba lo siguiente: “...no hay mayor amenaza para el medio ambiente que la demagogia, es decir, el engaño a los ciudadanos, el ocultismo intencionado de datos y decisiones, la manipulación interesada de la situación real de los recursos naturales y de las alternativas que existen para explorarlos adecuadamente" (World Resources Institute, 2004). El Informe concluía: "How we decide and who gets to decide often determines what we decide" (World Resources Institute, 2004). En general, los indicadores ambientales intentan satisfacer dos propósitos concretos: a) son un instrumento útil para la planificación de la sostenibilidad y el desarrollo de políticas orientadas en este sentido, y b) constituyen una valiosa herramienta de comunicación, con el fin de 
orientar las conductas en el sentido de la sostenibilidad. Los indicadores han de ser especialmente útiles para el seguimiento y evaluación de las actuaciones, de acuerdo con objetivos previamente establecidos. Han de ser, además, comprensibles y expresar de manera más asequible la información que como lo hacen los complejos índices estadísticos u otras fuentes de datos. Esto es, los indicadores ambientales, como cualquier otro indicador, proporcionan una información que va más allá de la cifra que suministra. Su finalidad principal es conducir a la acción. Éste es un aspecto fundamental, aunque no suela aparecer de manera explícita en la literatura.

Los indicadores ambientales, asimismo, difieren de otras medidas de información estadística, en cuanto a que constituyen un elemento de un proceso de acción o control específico. Son diseñados específicamente para aquellos procesos con los que están relacionados (Bakkes et al., 1994). El indicador es definido como un componente del proceso de información que: (i) es una parte de un proceso de gestión específico y puede ser comparado con los objetivos de dicho proceso, y (ii) al cual se le asigna "un significado más allá de su propio valor numérico" (Bakkes et al., 1994: 5). Así pues, del mismo modo que las actuales cuentas económicas persiguen propósitos concretos y diseñan indicadores adecuados a ellos, la contabilidad bajo la perspectiva de la sostenibilidad debe disponer de su propio sistema de indicadores (Cano-Orellana, 2004).

Las funciones de los indicadores en un contexto de adaptación eco-sistémica pueden concretarse en las siguientes: (i) importancia intrínseca: especies económicas, especies amenazadas, otras especies de importancia directa para los seres humanos; (ii) indicadores de alerta; (iii) indicadores de sensibilidad; (iv) indicadores de proceso; (v) indicadores de sensibilidad/vulnerabilidad ecosistémica.

Algunos de los criterios que podrían establecerse para la elección de indicadores podrían ser: (i) territorial, (ii) sectorial, (iii) fuentes o recursos para la gestión de conflictos, y (iv) posibilidad de elegir entre diferentes indicadores. Además de considerar aspectos como: (i) relevancia política y utilidad para los usuarios, (ii) consistencia analítica, y (iii) mensurabilidad.

Miller (2007) elaboró para el Instituto Internacional para el Desarrollo Sostenible (IISD, por sus siglas en inglés) un informe por encargo de 
la División de Naciones Unidas para el desarrollo sostenible (UNCSD, por sus siglas en inglés) con el propósito de poner al día los indicadores de desarrollo sostenible, tendencias futuras y una serie de claves y sugerencias para seguir avanzando en esta línea. Un par de años más tarde el autor reflexiona sobre los indicadores de sostenibilidad o de desarrollo sostenible desde una perspectiva social. En Creating indicators for sustainability. A social approach (Miller, 2007) se formula la siguiente pregunta: ¿cómo definimos el conjunto de medidas e indicadores? Se trata de discutir por qué una colectividad desea establecer indicadores de sostenibilidad, cómo puede llevarse a cabo la tarea de su identificación y elaboración y qué criterios deberíamos usar para evaluar los resultados. El propone un conjunto de criterios para la obtención de buenos indicadores, a saber: (i) relevancia política, (ii) sencillez de cálculo, (iii) validez científica, (iv) generador de series temporales, (v) disponibilidad y asequibilidad de datos, (vi) capacidad para agregar información, (vi) sensibilidad ante los cambios, (vii) fiabilidad, (viii) representatividad, (ix) utilidad, (x) comparabilidad, y (xi) razonable relación costos-beneficios. A estos criterios habría que añadir otros para dar cabida al componente social, entre ellos: 1) significativo para la sociedad, 2) buen gobierno, 3) conocimientoslocales, 4) peso histórico, 5) adaptabilidad y flexibilidad, y (6) generación de conocimiento.

Adicionalmente, los indicadores han de satisfacer, debido a las funciones que tienen encomendadas, una serie de requisitos tales como: (i) fáciles de entender y buena herramienta de comunicación, (ii) científicamente solventes, (iii) enviar señales inequívocas, (iv) aplicables a un buen número de variables, (v) cuantificación y desagregación, (vi) informar de la realidad de manera transparente, (vii) mejorar las fuentes de datos, (viii) visibilidad, (ix) disponibilidad para el análisis del ciclo de vida de los productos y los procesos, y (x) compatibles con las cuentas nacionales.

Los indicadores sostenibles también persiguen (PASTILLE, 2002): (a) entender la sostenibilidad, (b) servir de soporte para la toma de decisiones, (c) ayudar a la gestión, (d) empoderar e implicar a las partes, y (e) favorecer una adecuada resolución de conflictos entre intereses enfrentados.

Entre sus posibles aplicaciones podríamos indicar las siguientes: (i) evaluación, (ii) integración de aspectos ambientales en la toma de decisiones, 
(iii) divulgación, (iv) protección y mejora del medio ambiente, (v) seguimiento, (vi) predicción, (vii) comparación a nivel internacional, y (viii) herramientas para el conocimiento científico.

En resumen, los indicadores, las metas y los objetivos que deben alcanzarse son como puntos que pueden ser trazados y puestos en relación con el uso de un compás. Si se toman como centro los principios o criterios que rigen los contenidos de unos y otros es posible poner en conexión ambos extremos; esto es, los indicadores de un lado y las metas y objetivos de otro.

Los indicadores, en consecuencia, han de estar estrechamente vinculados a los objetivos perseguidos. Si transitamos desde un modelo convencional de sostenibilidad "three-ring circus" (tres anillos enlazados) -como sugiere de manera gráfica Levett (1998)- a otro más acorde con la realidad existente, como "russian dolls" (muñecas rusas o círculos concéntricos), los indicadores o baterías de indicadores que generemos han de operar el mismo tránsito. Esto es, el carrusel convencional de la producción y consumo, circunscrito al universo de los valores monetarios, se verá inscrito en una realidad más amplia, que para ser interpretada adecuadamente requerirá de instrumentos más afines a esta nueva perspectiva. Es la relación inclusiva propuesta por Passet (1996), que supone un cambio copernicano en la manera de entender la economía y su vinculación con la realidad más amplia, que constituyen los ecosistemas terrestres. Así, la actividad económica queda inscrita en otra más amplia, la actividad social y ésta, a su vez, en una aún mayor, la biosfera.

Así mismo, Hezri y Dovers (2006) consideran que los avances que se puedan alcanzar, en la teoría y en la práctica, en la consolidación de indicadores de sostenibilidad, dependerán de su permanente integración conceptual, metodológica e institucional. Los criterios, requisitos y recomendaciones facilitadas en los párrafos precedentes pueden facilitar la discusión en torno de ellos y su elección. ${ }^{11}$

${ }^{11}$ Puede consultarse también; Indicators of progress: Guidance on measuring the reduction of disaster risks and the implementation of the Hyogo framework for action, en International Strategy for Disaster Reduction (ISDR), United Nations Secretariat of the International Strategy for Disaster Reduction (UN/ISDR), Ginebra, enero, 2008. 


\section{Cuadro 4}

Principales aproximaciones al desarrollo de indicadores de sostenibilidad

\begin{tabular}{|l|l|l|}
\hline \multicolumn{1}{|c|}{ Temas } & \multicolumn{1}{|c|}{ Ejemplos de indicadores } & \multicolumn{1}{|c|}{$\begin{array}{c}\text { Estudios publicados en } \\
\text { EEJ }\end{array}$} \\
\hline $\begin{array}{l}\text { Extensión a las cuentas } \\
\text { nacionales }\end{array}$ & $\begin{array}{l}\text { Marco para la contabilidad } \\
\text { ambiental, ahorro auténtico } \\
\text { neto (adjusted net savings) } \\
\text { genuino indice de progreso } \\
\text { (GPI), el indice de bienestar } \\
\text { económico sostenible }\end{array}$ & $\begin{array}{l}\text { El Serafy, 1997; Hamilton, } \\
\text { 1999; Hueting and } \\
\text { Reijnders, 2004; Pearce } \\
\text { and Atkinson, 1993 }\end{array}$ \\
\hline Contabilidad biofísica & Huella ecológica & $\begin{array}{l}\text { Van den Bergh and } \\
\text { Verbruggen, ack 1999; } \\
\text { Wackernagel and Rees, } \\
\text { 1997 }\end{array}$ \\
\hline Índices ponderados & $\begin{array}{l}\text { Índice de Desarrollo } \\
\text { Humano (IDH) y otros } \\
\text { índices }\end{array}$ & $\begin{array}{l}\text { Fearnside, 2002; Morse, } \\
\text { 2003a; Neumayer, 2001 }\end{array}$ \\
\hline $\begin{array}{l}\text { Eco-eficiencia y } \\
\text { desmaterialización }\end{array}$ & $\begin{array}{l}\text { Flujos de recursos y y y } \\
\text { materiales }\end{array}$ & $\begin{array}{l}\text { Dellink and Kandelaars, } \\
\text { 2000; Hinterberger et al., } \\
\text { 1997 }\end{array}$ \\
\hline Batería de indicadores & $\begin{array}{l}\text { Lista de indicadores e } \\
\text { informes sobre el estado del } \\
\text { medio ambiente }\end{array}$ & $\begin{array}{l}\text { Azar } \text { et al., 1996; Friend } \\
\text { and Rapport, 1991; } \\
\text { Gustavson } \text { et al., 1999 }\end{array}$ \\
\hline
\end{tabular}

Fuente: Hezri y Dovers (2006).

La preocupación por la puesta al día del avance o transformaciones habidas en el ámbito de la generación de indicadores de sostenibilidad y su vinculación con las cuentas nacionales es permanente ${ }^{12}$, en consonancia con la presencia cada vez más importante de eventos, foros, investigaciones, etc., relativas a este ámbito de análisis, así como su papel en cuanto a los procesos de evaluación integrada, una cuya referencia fue la celebración en 1998 de la conferencia de apertura del Foro europeo sobre evaluación medioambiental integrada (Toth, 2003). En este mismo sentido, podemos reseñar la existencia de International Sustainability Indicators Network, que tiene habilitada una red para quienes están interesados en el diseño y la discusión, así como en el conocimiento de la situación de los indicadores de sostenibilidad.

$\overline{{ }^{12} \text { Una aplicación }}$ concreta de cómo se materializa este tipo de preocupaciones puede apreciarse en Pittman y Wilhelm (2007), González, Martín y Miguelez (2007). 
En Génova (Italia) a finales de agosto de 2010 tuvo lugar un encuentro organizado por el grupo de trabajo sobre evaluación y gestión medioambiental, junto con la Agencia europea de medioambiente y otras organizaciones internacionales, patrocinado por la División Estadística de Naciones Unidas (UNSD por sus siglas en inglés) y la Comisión Económica para Europa de Naciones Unidas (UNECE), con los siguientes objetivos: (a) discutir las prioridades y modalidades de cooperación entre expertos en estadística y medio ambiente durante 2009-2010, (b) revisar detalladamente los indicadores contenidos en la Guía para la aplicación de indicadores ambientales, (c) intercambiar información sobre los desarrollos a nivel internacional de indicadores ambientales, (d) iniciar las discusiones sobre los formatos actualizados de presentación y herramientas para una difusión eficaz de los indicadores ambientales.

En definitiva, si bien es cierto que se han producido avances notables no sólo en el diseño y generación de un buen número de indicadores ambientales destinados a diferentes propósitos, así como en la construcción de indicadores sintéticos de sostenibilidad, todavía es escasa en la literatura y, aún más, en las agendas públicas la puesta en marcha de una batería de indicadores que sirvan de soporte y, a su vez, se nutran de los nuevos sistemas contables. Tal vez los esfuerzos que se están desarrollando en este ámbito por diferentes organismos y las modificaciones derivadas de las recomendaciones internacionales ya establecidas, como se ha indicado en este trabajo, coadyuven a avanzar de manera definitiva hacia la necesaria convergencia de indicadores ambientales y las cuentas nacionales bajo la perspectiva de la sostenibilidad.

\section{CONSIDERACIONES FINALES}

El propósito fundamental perseguido en este artículo ha sido revisar cuáles han sido los modificaciones y mejoras que han tenido lugar en las cuentas nacionales, los sistemas contables públicos, así como de los indicadores asociados, desde una perspectiva sostenible, en el marco de la economía ecológica y de acuerdo con los criterios de gobernanza. Así como hacer una propuesta de reflexión acerca del papel de las cuentas nacionales y los indicadores ambientales, como herramientas para afrontar 
con ciertas garantías de éxito los retos a los que nos enfrentamos. Como hemos reiterado a lo largo del artículo la conciencia sobre la existencia de una relación especialmente conflictiva entre las prácticas humanas y el medio que nos sirve de soporte y sustento ha conducido a una situación insostenible. Los desarreglos sociales y ambientales están tan acentuados que la demora en ponernos en marcha constituiría un ejercicio de absoluta irresponsabilidad. Efectivamente, no sólo la conciencia social de tales problemas ha mejorado, también las diferentes agendas recogen cada vez con mayor profusión iniciativas que se orientan en esta dirección. Todo esto hay que interpretarlo como una buena noticia, sin embargo, estamos conscientes de que las inercias pesan mucho, que las mentalidades no cambian fácilmente, que requieren tiempo. Adicionalmente, es conveniente observar que el principal escollo que hay que salvar está relacionado con las mentalidades. Como sugiere Naredo (1987), el principal obstáculo a que se enfrentan los economistas es el instrumental conceptual y metodológico que utilizan. O como afirmara Hicks (1979), tan estrecho es el campo de los fenómenos con los que trabaja la economía que los economistas están continuamente dándose cabezazos contra sus propios límites. Esta visión tan poco realista se ve reflejada, sobre todo, en los sistemas contables. Por ello, tiene razón Naredo (1987) cuando afirma que la reducción practicada en el ámbito de lo económico se ve reflejada, ineludiblemente, en sus sistemas de contabilidad nacionales. En consecuencia, el problema fundamental al que tenemos que hacer frente es el de generar las condiciones para que se produzca ese tránsito deseado desde una economía que esté regida, de manera prioritaria, por el universo de los valores monetarios hacia aquella otra donde lo dominante sea la gestión y administración prudente de los bienes; así como orientada a procurar el mayor estado de bienestar posible al conjunto de la población. Para ello, éste es nuestro interés, es necesario engrasar el engranaje conceptual y metodológico estándar que, en ocasiones, representa más un obstáculo que una vía para aprehender mejor los fenómenos económicos y poder actuar, en consecuencia, con ciertas garantías de éxito. Se han producido avances, pero aún son insuficientes, por encima de todo es necesario una mayor coordinación y una voluntad decidida para, al menos, poner en funcionamiento aquellas herramientas que ya han sido diseñadas. Los sistemas contables deben 
ser más permeables a los cambios, los indicadores, por su parte, más eficaces; la relación entre ambos es imprescindible. Dado que los sistemas contables informan sobre el estado de la economía y posibilita la toma de decisiones, a través de la información directamente disponible o de los indicadores o índices generados a partir de ella, si ampliamos el campo de los fenómenos a que ella se enfrenta, los cuadros contables e indicadores habrán de adaptarse a este nuevo enfoque. Tal vez se haya avanzado más en el ámbito de los indicadores. Sin embargo, el problema suele ser, a veces, la difícil integración que éstos encuentran en el marco actual de las cuentas nacionales. Como se ha dicho en alguna ocasión, informan y reciben información de las cuentas satélites, pero el núcleo de la toma de decisiones más importante sigue dependiendo, principalmente, de las cuentas nacionales. Lo deseable sería transitar hacia una relación menos asimétrica, es decir, a que los satélites dejen de serlo y sean considerados como planetas. Éste debe ser nuestro empeño, nuestro reto. 


\section{REFERENCIAS}

Azqueta, D., G. Delacámara, M. Santamaría y S. Tirado. 2004. Contabilidad nacional y medio ambiente, Fundación de las Cajas de Ahorro, Madrid.

Bakkes, J., A. et al. 1994. An overview of environmental indicators: State of the art and perspectives. UNEP and RIVM, Bilthoven (NH).

Bartelmus, P. 1989. Environmental accounting and the system of national accounts, in Ahmad, Y., S. El Serafy and E. Lutz (eds.), Environmental accounting for sustainable development. Washington, D.C.: World Bank.

Bartelmus, P., C. Stahmer, and J. van Tongeren. 1991. Integrated environmental and economic accounting: framework for a SNA Satellite System, in Review of income and wealth, 37: 111-148

Cano-Orellana, A. 2000. "Indicadores de sostenibilidad. La necesidad de indicadores de síntesis", Economía y Medio Ambiente, Instituto de Estadística de Andalucía. Sevilla, 1: 159-175.

2004. Economía y sostenibilidad en las grandes aglomeraciones urbanas. Una aproximación al cálculo de la huella ecológica del área metropolitana de Sevilla, Sevilla Global.

2008. Indicadores territoriales de sostenibilidad: obstáculos, nuevas propuestas, en XI Jornadas de Economía Crítica. Bilbao, 2728 y 29 de marzo.

2009. "Territorio y sostenibilidad. Aproximación a la Huella Ecológica de Andalucía”, Revista de Estudios Regionales, 84: 115145 .

Carson, C. S. 1989. The United Nations System of National Accounts: a revision for the 21st century, documento presentado a la American Economic Association, Atlanta, 29 de diciembre. 
CEPAL 2005. Cuentas ambientales: conceptos, metodologías y avances en los países de América Latina y el Caribe, SERIE Estudios Estadísticos y Prospectivos, núm. 30.

Dietz, S., Neumayer, E. 2007. Weak and strong sustainability in the SEEA: concepts and measurement, Ecological Economics, 61 (4). pp. 617-626.

Environment Canada. 1991. A report on Canada's progress towards a national set of environmental indicators, Environment Canada, Otawa. Canada. González-Laxe, F., F. Martín y F. Miguélez (2007). Relationship between the quantification systems of national accounting and indicators of sustainable development, in 47th Congress of the European Regional Science Association. París.

Hezri, A. A., Dovers, S. R. 2006. "Sustainability indicators, policy and governance: Issues for ecological economics”, Ecological Economics, 60: 86-99.

INEGI. 2003. Historia del Sistema de cuentas nacionales de México (1938-200o), México.

INEGI. 2006. Sistema de cuentas económicas y ecológicas de México, 1999-2004, <http: //www.inegi.org.mx/prod_serv/contenidos/ espanol/bvinegi/productos/derivada/economicas/medio\%20 ambiente/2004/SCEEM99_04.pdf>

INEGI. 2010. Sistema de cuentas económicas y ecológicas de México, 2003-2008, <http://www.inegi.org.mx/inegi/contenidos/espa$\mathrm{nol} /$ prensa/comunicados/scnm-ecologicas10.asp>

Kapp, W. 1995a. La ruptura ambiental: un desafío a las ciencias sociales, en F. Aguilera (comp.) Economía de los recursos naturales: un enfoque institucional, Fundación Argentaria, Visor, Colección economía y naturaleza. 
Kapp, W. 1995b. "Los indicadores ambientales como indicadores de los valores sociales de uso", en F. Aguilera (comp.) Economía de los recursos naturales: un enfoque institucional, Fundación Argentaria, Visor, Colección economía y naturaleza.

Kapp, W. 2006. Los costes sociales y la empresa privada, Los Libros de la Catarata, Madrid.

Levett, R. 1998. Sustainability indicators - integrating quality of life and environmental protection, Journal of the Royal Statistical Society, Series A (Statistics in Society), 161(3): 291-302.

Miller, C.A. 2007. Creating indicator of sustainability. A social approach, International Institute for Sustainable Development, NY, mimeo.

Morilla, C.R. 2004a. Sistema híbrido para el análisis de las relaciones entre el medio ambiente, la economía y la sociedad. Aplicación para año 200o, al recurso agua y las emisiones a la atmósfera en España, tesis doctoral, Universidad de Sevilla.

Morilla, C.R. y G. Llanes. 2004b. "Gasto público y eficiencia ecoambiental de la economía española”, Presupuesto y Gasto Público, 36(3): 189-215.

Naredo, J.M. 1987. La economía en evolución. Historia y perspectivas de las categorías básicas del pensamiento económico, Siglo Veintiuno, Madrid.

OCDE. 1993. OECD core set of indicators for environmental performance reviews, Environment Monographs, 83, <http://www.oecd. org $>$.

OCDE. 1994. OCDE Environmental indicators, Ginebra.

Passet, R. 1996. Principios de bioeconomía, Fundación Argentaria, Visor, Colección economía y naturaleza. 
PASTILLE. 2002. Indicators into action. A practitioner guide for improving their use at the local level, Promoting Action for Sustainability Through Indicators at the Local Level in Europe.

Pittman, J. y K. Wilhelm. 2007. New economic and financial indicators of sustainability, New Directions for Institutional Research, 134: $55^{-69 .}$

Rodenburg, E. 1992. Eyeless in Gaia. The state of global environmental monitoring. Washington, D.C.: World Resources Institute.

Stahmer, C. 2002. Social accounting matrices and extended input-output tables, < www.oecd.org >.

Stiglitz, J., A. Sen y J.P. Fitoussi. 2011. Mismeasuring our lives: Why GDP doesn't add up, The New Press, NY.

Stone, R. 1961. Input-output and national accounts, OCDE.

Toth, F.L. 2003. State of the art and future challenges for integrated environmental assessment, Integrated Assessment, 4(4):. 250-264.

UNEP. 2011. Towards a green economy: Pathways to sustainable development and poverty eradication, <www.unep.org/greeneconomy >.

United Nations. 2005. Global Asessment Report. Millennium Ecosystem Asessment. http://www.maweb.org/en/Global.aspx

2006. Report of the committee of experts con environmentaleconomic accounting. E/CN.3/2007/9. Economic and Social Council. Statistical Commission Thirty-eighth session. Provisional Agenda. 
2008. Indicator of progress: Guidance on measuring the reduction of disaster risks and implementation of the Hyogo framework for action, en International Strategy for Disaster Reduction (ISDR), Secretariat of the International Strategy for Disaster Reduction (UN/ISDR), Ginebra, enero.

World Resources Institute. 2004. World resources 2002-2004: Decisions for the Earth: Balance, voice, and power, <http://archive.wri. org/pubs/pubs_pdf.cfm?PubID=3764 >. 2004. Annual Report 2004: Ideas into action. Working at the intersection of environment and human needs. Http:/pdf.wri.org/ annual report_2004_full.pdf 